\title{
Neue standesrechtliche Regelung
}

\section{Caroline Hartmann}

Dr. iur., Rechtsanwältin, Co-Leiterin FMH-Gutachterstelle

Die FMH-Gutachterstelle hat ein neues Reglement geschaffen, welches am 1. Oktober 2019 in Kraft tritt [1]. Die Verteilung der Kosten ist für die beteiligten Parteien reglementiert. Für die Bezahlung der Honorare der Gutachter gibt es eine neue standesrechtliche Regelung. Der vorliegende Beitrag erläutert diese Regelung und zeigt deren Auswirkungen für FMH-Mitglieder.

Um den medizinischen Sachverhalt und damit die Frage der ärztlichen Sorgfaltspflichtverletzung zu klären, ist grundsätzlich ein medizinisches Gutachten notwendig. Die FMH führt seit 1982 eine aussergerichtliche Gutachterstelle zur Begutachtung von vermuteten ärztlichen Sorgfaltspflichtverletzungen. Ist der angeschuldigte Arzt FMH-Mitglied, so ist er auf der Grundlage des Ärztekammerbeschlusses vom 24. Juni 1993 verpflichtet, sich auf eine FMH-Begutachtung einzulassen [2].

\section{Leistungen der Berufshaftpflicht- versicherung im Schadenfall}

Bei allfälligen Ersatzansprüchen eines Patienten an seine behandelnde Ärztin bestehen die Leistungen der Berufshaftpflichtversicherung einerseits aus einer Entschädigung berechtigter Ansprüche, andererseits auch aus der Abwehr unberechtigter Ansprüche (passiver Rechtsschutz).

Die Leistungen der Berufshaftpflichtversicherung bestehen in der Entschädigung berechtigter und der Abwehr unberechtigter Ansprüche.

Die Versicherung der Ärztin wird im Schadenfall abschätzen, ob sie für die Klärung des Sachverhalts ein medizinisches Gutachten als notwendig erachtet. Stimmt die Versicherung einer FMH-Begutachtung zur Klärung des Sachverhalts zu, wird auf der Grundlage der Berufshaftpflichtversicherungspolice eine Kostendeckung gegeben sein. Sei dies, da bei einer gutachterlichen Fehler- und Kausalitätsanerkennung die Kosten des Gutachtens Teil des Schadens bilden; oder sei dies im Rahmen des passiven Rechtsschutzes, d.h. zur Abwehr unberechtigter Ansprüche.

\section{Gentlemen's Agreement}

Das «Gentlemen's Agreement» besteht seit 37 Jahren zwischen der FMH und dem Schweizerischen Versicherungsverband (SVV). Versicherungen, die Mitglied des SVV sind, zahlen auf der Grundlage des «Gentlemen's Agreement» die Honorare der Gutachter vorbehaltlos, d.h. unabhängig vom Ergebnis des Gutachtens und unabhängig davon, ob die Versicherung eine FMH-Begutachtung als notwendig erachtet. ${ }^{1}$

Versicherungen, die Mitglied des SVV sind, zahlen die Honorare der Gutachter vorbehaltlos.

\section{Fehlende Kostendeckung}

Die Kostendeckung ist dann nicht garantiert, wenn der betroffene Versicherer nicht Mitglied des SVV ist und im konkreten Fall keine Kostengutsprache erteilt. Wegen fehlender Kostendeckung konnte die FMHGutachterstelle in der Vergangenheit kein Gutachten in Auftrag geben. Der Patientin steht es in solchen Fällen dann offen, auf eigene Kosten ein Privatgutachten in Auftrag zu geben oder den gerichtlichen Weg einzuschlagen. Wenn immer möglich sollten gerichtliche Verfahren aber vermieden werden, da diese geprägt sind von Beweisschwierigkeiten, die Parteien das Kostenrisiko tragen und aufgrund der langen Prozessdauer immer auch eine Negativbelastung darstellen.

\section{Art. 35 Abs. 2 Standesordnung FMH}

FMH-Mitglieder werden neu aufgrund des Ärztekammerbeschlusses vom 9. Mai 2019 vereinsrechtlich ver- 
2 Die bei der FMH-Gutachterstelle meist betroffenen Fachdisziplinen sind die Chirurgie, die orthopädische Chirurgie und Traumatologie des Bewegungsapparates, die Allgemeine Innere Medizin (inkl. Hausarztmedizin) und die Gynäkologie und Geburtshilfe. Die FMCH hat einen Rahmenvertrag mit der Basler

Versicherung AG (Mitglied des SVV), damit auch

chirurgisch und invasiv

tätige FMH-Mitglieder mit höheren Risiken für eine mögliche Sorgfaltspflichtverletzung eine für sie entsprechende Berufshaftpflichtversicherung abschliessen können.

3 Eine medizinische Vorprüfung wird durch die jeweilige medizinische Fachgesellschaft vorgenommen.

Aussergerichtliche Gutachterstelle der FMH Postfach 65

CH-3000 Bern 15 pflichtet, die Honorare der Gutachter zu übernehmen, wenn ihre Berufshaftpflichtversicherung nicht Mitglied des SVV ist und im konkreten Fall eine Kostengutsprache verweigert:

\section{Art. 35 Abs. 2 (neu) Standesordnung}

"Arzt und Ärztin sind verpflichtet, sich auf eine Begutachtung der FMH-Gutachterstelle einzulassen. Sofern keine Kostengutsprache durch den Haftpflichtversicherer erfolgt, ist der Arzt oder die Ärztin verpflichtet, das Honorar des Gutachters des von der FMH-Gutachterstelle in Auftrag gegebenen Gutachtens zu übernehmen.»

\section{Geringes Kostenrisiko für FMH-Mitglieder}

Das Kostenrisiko für FMH-Mitglieder ist trotz der neuen standesrechtlichen Regelung gering. Die meisten Berufshaftpflichtversicherer sind Mitglied beim SVV und damit an das "Gentlemen's Agreement" gebunden. ${ }^{2}$ Dem SVV sind rund 80 kleine und grosse, national und international tätige Versicherer angeschlos$\operatorname{sen}[3]$.

\section{Das Kostenrisiko für FMH-Mitglieder ist gering.}

Nicht alle Beanstandungen von Patienten führen zu einem FMH-Gutachten. Aussichtslose Fälle werden bereits durch die Patientenanwälte und Patientenanwältinnen sowie die Patientenstellen nicht weiterverfolgt. Zudem tritt die FMH-Gutachterstelle nur auf diejenigen Fälle ein, welche genügend Anhaltspunkte einer ärztlichen Sorgfaltspflichtverletzung zeigen. ${ }^{3}$

\section{Nicht alle Beanstandungen von Patienten führen zu einer FMH-Begutachtung.}

Zu erwähnen bleibt, dass FMH-Mitglieder zwar der Einlassungspflicht für eine FMH-Begutachtung unterstehen, die Parteien aber frei sind, sich auf eine andere Gutachterstelle zu einigen.

\section{Inkrafttreten von Art. 35 Abs. 2 Standes- ordnung FMH - Übergangsfrist}

Die neue Regelung von Art. 35 Abs. 2 Standesordnung FMH wird per 1. April 2020 in Kraft treten. Mit diesem Aufschub des Inkrafttretens wird eine Übergangsfrist gewährt. Diese erlaubt es Ihnen, entsprechende Abklä- rungen bei Ihrer Berufshaftpflichtversicherung zu tätigen und allenfalls Anpassungen im Versicherungsschutz vorzunehmen.

\section{Art. 35 Abs. 2 Standesordnung FMH wird} per 1. April 2020 in Kraft treten.

\section{Berufshaftpflichtversicherung \\ Die FMH empfiehlt eine regelmässige Kontrolle Ihrer Haftpflicht- versicherungspolice. Dabei stellen sich folgende Fragen \\ - Haben Sie eine Versicherungssumme von mind. 10 Millionen Franken? \\ - Sind alle Ihre ärztlichen Tätigkeiten versichert? \\ - Haben Sie eine Nachversicherung bei Geschäftsaufgabe während der gesetzlichen Verjährungsfristen? \\ - Ist Ihre Berufshaftpflichtversicherung Mitglied beim SVV?}

Eine Beratung erhalten Sie bei FMH Insurance Services [4].

\section{Schlussfolgerung}

Nicht alle Berufshaftpflichtversicherungen, die in der Schweiz tätig sind, sind Mitglied beim SVV, weshalb sie nicht an das «Gentlemen's Agreement» gebunden sind. Diese Versicherungen prüfen zwar eine Kostenübernahme von Fall zu Fall, weigern sich aber erfahrungsgemäss bei einer Begutachtung, die Kosten vorbehaltlos zu übernehmen.

Besteht weder auf der Grundlage der Haftpflichtversicherungspolice eine Kostendeckung für das Gutachterhonorar, noch ist die betroffene Berufshaftpflichtversicherung Mitglied beim SVV und damit nicht an das «Gentlemen's Agreement» gebunden, kommt der neue Art. 35 Abs. 2 Standesordnung FMH zur Anwendung. Gemäss dieser Bestimmung ist der betroffene Arzt oder die betroffene Ärztin als FMH-Mitglied verpflichtet, das Honorar des Gutachters des von der FMHGutachterstelle in Auftrag gegebenen Gutachtens zu übernehmen.

Mit einer entsprechenden Versicherungslösung kann jedoch das Kostenrisiko auf die Versicherung abgewälzt werden.

Literatur

1 Vgl. Hartmann C. Neues Reglement der aussergerichtlichen FMHGutachterstelle. Schweiz Ärzteztg. 2019;100(38):1252-3.

2 Art. 35 Standesordnung FMH.

3 Vgl. die Übersicht der Mitglieder des SVV unter www.svv.ch/de/ die-mitglieder-des-svv, besucht am 28.8.2019.

4 Vgl. www.fmhinsurance.ch, Roth Gygax \& Partner AG, Moosstrasse 2, 3073 Gümligen. 